\title{
Data Security Accessing for HDFS Based on Attribute-Group in Cloud Computing
}

\author{
Huixiang Zhou \\ State Key Laboratory of Networking and Switching \\ Technology, BUPT \\ Beijing, China \\ Zhouhuixiang168@163.com
}

\author{
Qiaoyan Wen \\ State Key Laboratory of Networking and Switching \\ Technology, BUPT \\ Beijing, China \\ wqy@bupt.edu.cn
}

\begin{abstract}
To solve the security issues of network features and data sharing features in cloud storage service, this paper proposes a data security access scheme in cloud storage Based on Attribute-Group. In terms of access rights control and access control architecture for the access control mechanism research, in this scheme, data owners do not participate in the specific operation of the property and user rights, re-encryption on the NameNode, can reduce the cost of the computation and management of the client, and also reduce the complexity of rights management and property management.
\end{abstract}

Key words: cloud storage, data security access, Hadoop, HDFS, attribute-Group.

\section{INTRODUCTION}

Research of IDC shows that from 2007 to 2012, more than 6 times the global growth in the amount of information, from 161EB increased 988EB (1EB = $1024 \mathrm{~PB})$. The face of petabytes of mass storage needs, there is bottleneck in the expansion of capacity and performance on the traditional SAN or NAS, and even some of the existing high-end storage devices obviously cannot meet this demand [1]. The mass storage demand for storage capacity, storage access performance and cost of the unprecedented challenges, cloud storage will become an ideal way for future storage because of its superior scalability.

However, whether the user be assured that put $t$ he private data to various cloud is the key that whet her cloud storage can be widely promoted. And the user's sense of trust cannot simply rely moral constra ints or rules on the management and finally be resol ved. If the technically can makes private data only $b$ e looked by its own, that can fundamentally solve th e problem that user trust in the cloud storage.

Key corresponds to a set of attributes, based on the group attributes encrypted cipher text strategy (Cipher text-policy Attribute-based Encryption, CP-ABE)[4] cipher text corresponds to an access structure and decrypted if and only if the set of attributes property to meet the access structure. CP-ABE applies the decrypting party fixed in a distributed environment, the decryption rule contains the encryption algorithm, $\mathrm{CP}-\mathrm{ABE}$ this particular integrated multi-NameNode characteristics suitable for cloud storage, which can be removed from the cipher text access control that occur frequently in the cost of key distribution. Apache Hadoop[5] is an open-source software framework that supports data-intensive distributed applications, the Hadoop Distributed File System (HDFS)[6] is a distributed, scalable, and portable file system written in Java for the Hadoop framework, and HDFS is cloud storage the most widely used tool. This article is based on the $\mathrm{CP}-\mathrm{ABE}$ and HDFS designing a secure cloud storage the cipher text control program. On the basis of the CP-ABE and symmetric encryption algorithm (such as AES), we propose a cloud-oriented storage efficient dynamic access control scheme cipher text.

\section{RELATED RESEARCH}

\section{A. HDFS technology}

HDFS is a distributed, scalable, and portable file system written in Java for the Hadoop framework. Each node in a Hadoop instance typically has a single NameNode, a cluster of DataNodes form the HDFS cluster. The situation is typical because each node does not require a DataNode to be present. Each DataNode serves up blocks of data over the network using a block protocol specific to HDFS. The file system uses the TCP / IP layer for communication. Clients use Remote procedure call (RPC) to communicate between each other. HDFS stores large files (an ideal file size is a multiple of $64 \mathrm{MB}$ ), across multiple machines. It achieves reliability by replicating the data across multiple hosts, and hence does not require RAID[7]b5 storage on hosts. With the default replication value , 3 , data is stored on three nodes: two on the same rack, and one on a different rack. Data nodes can talk to each other to rebalance data, to move copies around, and to keep the replication of data high. HDFS is not fully POSIX compliant, because the requirements for a POSIX file system differ from the target goals for a Hadoop application. The tradeoff of not having a fully POSIX-compliant file system is increased performance for data throughput and support for non-POSIX operations such as append.

\section{B. $C P-A B E$}

Sahai and Waters first proposed based on fuzzy identity is encrypted[8], as the identity of the biological characteristics apply the information based on the concept 
of the identity of the encryption scheme, Sahai introduced in the paper attributes, based on 2006, Goyal et al The fuzzy identity encryption scheme based on the attribute-based encryption scheme (ABE, attribute-based encryption). 2007, Bethencourt et al cipher text policy attribute-based encryption (CP-ABE, cipertext-policy $\mathrm{ABE}$ ), the user's identity is represented as a collection of a property, and encrypt data access control structure Union, whether a user can decrypt the cipher text, depending on whether the cipher text associated set of attributes corresponding to the user identity-based access control structure match.

\section{CPE-ABE algorithm}

Attributes: Set $P=\left\{P_{l}, P_{2}, \mathrm{~L}, P_{n}\right\}$ collection of all properties, properties for each user $\mathrm{A}$ is a non-empty subset of $\mathrm{P}, A \in 2^{N}$, then the $\mathrm{N}$ attribute can be used to identify $2^{N}$ user.

Access structure: Access structure $\mathrm{T}$ is a nonempty subset of the complete works $\left\{P_{l}, P_{2}, \mathrm{~L}, P_{n}\right\}$ and $T \in 2^{\left\{P_{l}, P_{2}, \mathrm{~L}, P_{n}\right\}} \quad A \in\left\{P_{l}, P_{2}, \mathrm{~L}, P_{n}\right\} \backslash\{\phi\}$. T represents an attribute judgment condition: in $\mathrm{T}$ attribute set is called the authorization set, not in the $\mathrm{T}$ attribute set is called the set of non-authorized.

\section{SCHEME OF DATA SECURITY ACCESS BASED ON ATTRIBUTE-GROUP}

In this paper, the properties of the cipher text $\mathrm{CP}-\mathrm{ABE}$ encryption algorithm based on cloud storage data security access control scheme. Compared to the data owner directly distributed key distribution, centralized management of key distribution method and NameNode-based CP-ABE, easier to manage keys, but also more transparent to the user, that allows users to less involved key generation, key distribution, and other matters.

There is certain credibility, requiring CSP must be faithful to run the program and visits

Asked the agreement, yet may spy the contents of the data file, and assumes that all parameters and between the communication channel is secure.

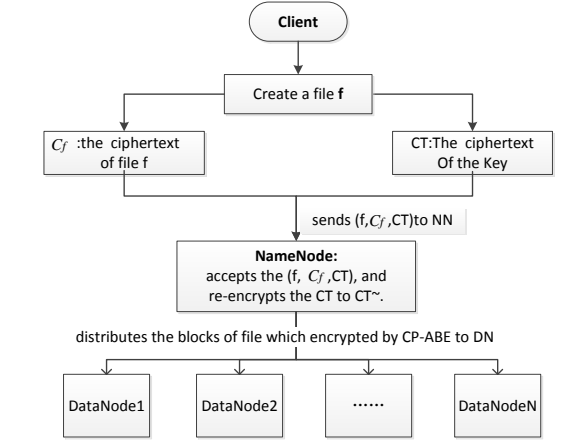

Fig1. the architecture of Data security access Scheme

\section{A. System Initialization}

The trusted authority CA 2 of order the primes p cyclic group $G_{1}$ and $G_{2}$ bilinear mapping $e: G_{1} \times G_{(} \rightarrow G_{2}$ g is the $G_{1}$ generator. Define the attribute space $\lambda=\left\{\lambda_{1}, \lambda_{2} \mathrm{~L}, \lambda_{q}\right\}$, each attribute $\lambda_{i} \in \lambda(1 \leq i \leq q)$, randomly selected $w_{i} \in G_{l}, \operatorname{att}\left(\lambda_{i}\right)$ return the property $\lambda_{i}$ corresponding ID i, $U=\left\{u_{l}, u_{2}, \mathrm{~L}, u_{n}\right\}$ all users in the system, the attribute group $U \lambda_{i} \subset U$ property $\lambda_{i}$ set of users. The $\mathrm{CA}$ randomly selected $\alpha, \beta \in Z_{\rho}^{*}$ publish the public parameters $\left.g, g^{\beta}, e(g, g)^{\alpha},\left\{w_{i}\right\}_{i=l}^{J}\right\}$, kept secret master key $M K=\left(\beta, g^{\alpha}\right)$.

\section{B. Key generation}

The key generation include two parts: the user's private key generation algorithm and path key generation algorithm.

1) The user's private key generation algorithm:

Enter the user set $U$ and set of attributes lambda, the algorithm outputs each user $u_{t} \in U$ set of attributes $\Lambda \subset \lambda$ of the corresponding private key $S K_{t}$. CA select $r \in Z_{\rho}^{*}$ randomly (corresponding to each user r), for each attribute $\lambda_{i} \in A$ randomly selected $\quad r \in Z_{\rho}^{*}$ contingent. After generating the private key of each, $S K_{t}=\left(D=g^{(\alpha+r) / \beta}, \forall \lambda_{i} \in A: D_{i}=g^{r}(w, l H(\cdot))^{r}, D_{i}=g^{i}\right)$ of user $u_{t}$.

Finally, CA via a secure channel $S K_{l}$ send $u_{t}$, each attribute $\lambda_{i}$ corresponding attribute group the $U \lambda_{i}$ send data administrator NameNode. Set of attributes of user $u_{l}, u_{2}, u_{3}$ and $u_{4}$ is $\left\{\lambda_{1}, \lambda_{2}\right\},\left\{\lambda_{2}, \lambda_{3}, \lambda_{4}\right\},\left\{\lambda_{1}, \lambda_{3}\right\}$, and $\lambda_{1}, \lambda_{12}, \lambda_{3}, \lambda_{4}$. CA property $\lambda_{1}, \lambda_{12}, \lambda_{3}, \lambda_{4}$ corresponding attribute group FF, $U \lambda_{1}=\left\{u_{l}, u_{3}, u_{4}\right\}$, $U_{\lambda_{2}}=\left\{u_{l}, u_{2}, u_{4}\right\}, U_{\lambda_{3}}=\left\{u_{2}, u_{3}, u_{4}\right\}$ and $U_{\lambda_{4}}=\left\{u_{2}, u_{4}\right\}$ sent to the NameNode.

2) Path key generation algorithm:

NameNode to construct a binary tree to (called KEK tree), where the leaf nodes of the tree members of each user in $\mathrm{U}, v_{j}$ assignment of each node in the tree a random number $K E K_{j} \in Z_{\rho}^{*}$. Corresponding to the root of the tree with the user $\boldsymbol{u}_{t}$ There is a path between the leaf node, $u_{t}$ path defined that all nodes on the path corresponding to the random number key $P K_{t}$.

The NameNode sents $P K_{t}$ to the user via a secure channel $u_{t} \in U$. KEK tree as shown in Fig1, the user sets $U=\left\{u_{l}, u_{2}, u_{3}, u_{4}, u_{5}, u_{6}, u_{7}\right\}$, the path of the user key $U=\left\{u_{l}, u_{2}, u_{3}, u_{4}, u_{5}, u_{6}, u_{7}\right\}$ of $u_{1}$.

\section{C. file creation}

Data owners do first create a data file $\mathrm{f}$, then randomly select a symmetric key $k_{f}$ using the symmetric 
encryption algorithm $\mathrm{E}$ and $k_{f}$ encryption $\mathrm{f}$ to data cipher text $U=\left\{u_{l}, u_{2}, u_{3}, u_{4}, u_{5}, u_{6}, u_{7}\right\}$. To construct an access tree $\mathrm{T}$, each leaf of the tree Node represents an attribute. $k_{\tau}$ set the threshold value of each node in the $\mathrm{T} \mathrm{x}$, node $\mathrm{x} q_{x}$ of a polynomial $k_{X}-1$.

Random selection $s \in Z_{\rho}^{*}$ to the root node $\mathrm{R}$, the $q_{R}(0)=s \quad, \quad$ For non-root node $\mathrm{x}$, the $q_{x}(0)=q_{\rho^{\prime \prime} \operatorname{rent}(X)}(\operatorname{index}(x))$ parent $(\mathrm{x})$ Returns the parent node of the node $\mathrm{x}$ index $(\mathrm{x})$ returns the serial number of the node $\mathrm{x}$ in its sibling. Assume that $\mathrm{Y}$ is a collection of all the leaf nodes of T, DO generate key cipher text of $k_{f}$ :

$C T=\left(T, C=k_{f} e(g, g)^{\alpha s}, C=g^{\beta s}, \forall y \in Y:\right.$

$\left.C_{y}=g^{4_{y}(0)}, C_{y}^{I}=\left(w, " t\left(\lambda_{J}\right)\right)^{4_{y}(0)}\right)$

Finally, do $\left(C_{f}, C T\right)$ sent along to the CSP.

\section{File re-encryption}

Data Services NameNode received $\left(C_{f}, C T\right)$, CT following the re-encryption operation:

1) For each attribute $y \in Y$ corresponding attribute group $U_{\lambda_{J}}$ randomly selected $K_{\lambda_{J}} \in Z_{\rho}^{*}$ of $U_{\lambda_{J}}$ group key calculation:

$$
\begin{aligned}
& C T^{\vee}=\left(T, \stackrel{\mathcal{C}}{C}=k_{f} e(g, g)^{\alpha s}, C=g^{\beta s},\right. \\
& \left.\forall y \in Y: C_{y}=g^{(0)}, C_{y}^{1}=\left(\left(w_{\text {att }\left(\lambda_{y}\right)}\right)^{q_{y}(0)}\right)^{K \lambda_{y}}\right)
\end{aligned}
$$

2) at the KEK tree, looking for the $U \lambda_{i}$ able to cover all users the best KID tree, and the root node of the sub tree corresponding to the random number is defined as the path of the attribute group key, denoted $\operatorname{KEK}\left(U_{\lambda_{i}}\right)$. For example, in Fig1, if the attribute group $U_{\lambda_{L}}=\left\{u_{l}, u_{3}, u_{4}\right\}$ KEK tree can override the user $u_{1}$, $u_{3}$ and $u_{4}$ minimal root of the tree $\{v 5, v 8\}, U_{\lambda_{L}}$ then the path key $\left\{K E K_{5}, K E K_{8}\right\}$, that is, only the $U_{\lambda_{L}}$ user permission to know $K E K\left(U_{\lambda_{L}}\right)$.

3) with a symmetric encryption algorithm E, and the path of the attribute group key to encrypt the group key, Generate the header message is as follows:

$$
H d r=\left(\forall y \in Y:\left\{E_{K}\left(K_{\lambda_{J}}\right)\right\}_{K \in K B K\left(U_{l}\right)}\right) \text { (4) }
$$

The data is stored in NameNode $\left(H d r, C_{f}, C T^{`}\right)$.

\section{E. File Access}

User access to the data file f: the decryption header message HDR group Key, decryption key secret text CT get symmetric key $k_{f}$, the decrypt, according to data cipher text $C_{f}$ data file f. Specifically described as follows:
1) Assuming the user the $u_{t}$ to a CSP initiated requests for access to data files $\mathrm{f}$, NameNode returns $u_{t}$ to $\left(H d r, C_{f}, C T^{\prime}\right) \mathrm{e}$.

2) The $u_{t}$ an attribute group corresponding group key decryption Hdr. We may assume that the user $u_{t}$ is a legitimate property $\lambda_{i}$, then $u_{t}$ to use $K E K \in K E K\left(U_{\text {var }}\right)$ I $P K_{t}$ decryption Hdr get the attribute group $U_{\lambda_{L}}$ corresponding group key $K_{\lambda_{l}}$.

If the attribute group $U_{\lambda_{L}}=\left\{u_{1}, u_{3}, u_{4}\right\}$ key due to the path of the attribute group aa12 path keys

$K E K(U \cdot)=\left\{K E K_{5}, K E K_{8}\right\}$ of $U_{\lambda_{L}}$, the user

$P K_{1}=\left\{K E K_{1}, K E K_{2}, K E K_{4}, K E K_{s}\right\}$ of

$u_{1}, P K_{4}=\left\{K E K_{1}, K E K_{2}, K E K_{5}, K E K_{11}\right\}$ of $u_{4}$. So the

$K E K_{8} \in K E K\left(U_{\lambda_{L}}\right)$ I $P K_{1}$ and $u_{4}$ use

$K E K_{5} \in K E K\left(U_{\lambda_{L}}\right)$ I $P K_{4}$ can be decrypted HDR attribute group. The $K E K_{5} \in K E K\left(U_{\lambda_{L}}\right) \mathrm{I} \mathrm{PK}_{4}$ the

corresponding group key $K_{\lambda_{L}} \cdot u_{t}$ update their private key as follows:

$S K_{t}=\left(D=g^{(\alpha+r) / \beta}, \forall \lambda_{i} \hat{\in}: D_{i}=g\left(w, l^{\prime \prime}(\lambda \cdot)\right)^{r_{i}}, D_{i}^{I}=\left(g^{r_{i}}\right)^{K_{j i}}\right)$

3) $u_{t}$ uses new private key to decrypt the text $\mathrm{CT}^{\top}$. For non-leaf node $\mathrm{x}$ Let $\mathrm{x}$ child node set $\left\{z_{j}\right\}, j \geq k_{x}$, calculated each child node $z_{j}$ corresponding

$F_{Z_{j}}=\operatorname{DecryptNode}\left(C T^{I}, S K, z_{j}\right)=e(g, g)^{r q_{Z_{j}}(0\}}$

, and then select the $k_{x}$ sub node $F_{z j}$ as Lagrange Interpolation the polynomial interpolation nodes $F_{x}=e(g, g)^{r q_{x}(0\}}$. For the root node $\mathrm{R}$, the $A=\operatorname{DecryptNode}(C T, S K, R)=e(g, g)^{r q_{R}(0)}=e(g, g)^{r s}$ . If the a $u_{t}$ property set lambda meet the access tree $\mathrm{T}$, the symmetric key $k_{f}=C /(e(C, D) / A)$.

4) $u_{t}$ uses $k_{f}$ decrypts the $C_{f}$ data express file $\mathrm{f}$.

\section{SECURITY ANALYSIS}

The new program has data confidentiality. Proof: data cipher text $C_{f}$ due to the symmetric key randomly generate $k f$ by the DO, $k_{f}$ for the attacker is a random number, the NameNode know attribute group key, but 'decryption CT $k_{f}$ face decipher CP-ABE cryptosystem, so the new program with the confidentiality of the data cipher text. Set of attributes because the attacker cannot meet the access tree $\mathrm{T}$, so cannot be calculated $e(g, g)^{\alpha s}$, and thus cannot be restored $k_{f}$, the property if the attacker does not have the property $\lambda_{i}$ Group $U \lambda_{i}$ group key $K \lambda_{i}$ the 
attacker is a random number, so the new program with key cipher text confidentiality.

If the attacker from the header message HDR group key, equivalent to decipher AES symmetric encryption system, to date, the AES and CP-ABE is a safe enough strength encryption algorithm, the new scheme has the header information Confidentiality. Shown in the foregoing, the new program has data confidentiality.

The new scheme has anti-collusion attack. Proof: we can see, the only attribute set to meet the access tree to calculate the $e(g, g)^{r s}$ (assuming a single unauthorized users access only part of the property to meet the tree, but several unauthorized users conspiracy attack by the construction of the access tree $\mathrm{T}$.

When attribute set to meet the access tree T. By the user's private key generation algorithm shows that $r$ corresponding to the different users, so each user's private key $D_{i}=g^{r}\left(W_{a t t}\left(\lambda_{i}\right)\right)^{r i}$ different from each other. The multiple unauthorized users can only be calculated for each $e(g, g)^{r q_{x}(0)}$ at the value of the corresponding node $\mathrm{x}$, but cannot get $e(g, g)^{r s}$, so that the attacker cannot decrypt the $k f$. As the group key for external users is a random number, unauthorized collusion attack cannot attribute group the group key. Therefore, the new scheme has anti-collusion attack.

Before the new program has to confidentiality and after to confidentiality. Proof: The privileges revoked, NameNode the cipher text component transformation of the secret $\mathrm{s}$ corresponding secret

$\mathrm{s}$ 'the corresponding cipher text assembly. When the user leaves the attribute group $U \lambda_{i}$, the user know that the old group key $K \lambda_{i}$, but do not know the group key update left $K_{i}^{\prime}$, s is a random number, so the user knows $e(g, g)^{a s}$, but the cipher text corresponding withdrawal of property $e(g, g)^{a\left(s+s^{\prime}\right)} \quad$ cannot be calculated, the new program has a forward secrecy. When a user joins the attribute group $U \lambda_{i}$, new users to know updated group key $K^{\prime} \lambda_{i}$, but do not know the group key $K \lambda_{i}$, randomness by s `shows new users to know $e(g, g)^{a\left(s+s^{\prime}\right)}$, but has close before the property cannot be calculated text corresponding $e(g, g)^{a s}$, so the new program has to confidentiality.

\section{CONCLUSIONS}

Cloud storage security issues affecting the development of cloud storage, reasonable and effective data security access control method can improve the trust of the users for cloud storage services. To solve the security issues of network features and data sharing features in cloud storage service, this paper proposes a data security access scheme storage Based on Attribute-Group in cloud, so that the data owners do not participate in the specific operation of the property and user rights, while the re-encrypted transfer to the NameNode-side, reduce the amount of computation and management costs of the client, ensure the confidentiality of user data, and also Achieve its purpose that the cipher text file sharing. Although the attribute-group-based scheme proposed in this paper has high security and reliability, but the efficiency of the implementation has yet to be improved which is our next step to research.

\section{ACKNOWLEDGMENT}

This work is supported by NSFC (Grant Nos. 61300181, 61272057, 61202434, 61170270, 61100203, 61121061), the Fundamental Research Funds for the Central Universities (Grant No. 2012RC0612, 2011YB01).

\section{REFERENCES}

[1] Hadoop.http://Hadoop.apaehe.org,2010.

[2] Qinyin91 L, Xiaolinl G, Deqin S, et al. Secure storage for cloud storage Strategy [J]. Journal of Computer Research and Development, 2011, 48: 240-243

[3] WANGLQ,ZHANG J,LVSW,etal.An efficient cryptosystem based hierarchical access control scheme and its analysis[J].Computer Engineering and Applications, 2005,33,7-10.

[4] RAYI,RAI,NARASIMHAMURTHIN.A cryptographic solution to

implement access control in a hierarchy and more[A].Proceedings of the Seventh ACM Symposium on Access Control Models and Technologies[C].Monterey, California, USA, 2007,5-16.

[5] BETHENCOURTJ,SAHAIA,WATERSB. Cipher text-policyattrib

ute-based encryption[A]. 2007 IEEE Symposiumon Security and Privacy(SP'07)[C]. Berkeley, California, USA. 2007.

[6] Borthakur D. The hadoop distributed file system: Architecture and design[J]. Hadoop Project Website, 2007, 11: 21.

[7] Liu X, Han J, Zhong Y, et al. Implementing WebGIS on Hadoop: A case study of improving small file I/O performance on HDFS[C]//Cluster Computing and Workshops, 2009. CLUSTER'09. IEEE International Conference on. IEEE, 2009: 1-8.

[8] Patterson D A, Gibson G, Katz R H. A case for redundant arrays of inexpensive disks (RAID)[M]. ACM, 1988. 